\title{
MULTIPLE ACTIVE CONTOUR MODELS BASED ON THE EM ALGORITHM $^{1}$
}

\author{
Margarida Silveira $^{(1)}$ Jorge S. Marques ${ }^{(2)}$ \\ ${ }^{(1)}$ IST/INESC, Lisbon, Portugal \\ ${ }^{(2)}$ IST/ISR, Lisbon, Portugal
}

\begin{abstract}
This paper describes an algorithm for the extraction of multiple regions using multiple active contour models (ACMs). The algorithm organizes edge points into strokes and assigns a set of weights summing to one to each stroke. These weights represent the soft assignment of the stroke to each of the ACMs and depend on the distance between the stroke points and the ACM units. Both the weights and the ACMs energy minimization are computed using the expectationmaximization (EM) algorithm. The algorithm described in this paper is an extension of the Adaptive Snakes recently proposed in [9]. Experimental results will be provided to illustrate the performance of the proposed algorithm.
\end{abstract}

\section{INTRODUCTION}

Active contour models (ACMs)[1] have been extensively used to estimate object boundaries in images. Active contours are influenced by external forces that push the contour towards image features such as edge points and by internal forces that try to keep the shape's continuity and smoothness.

Most of the research done on ACMs tries to estimate a single region using one elastic model (e.g. see [1][2][3]). The estimation of multiple elastic models has received much less attention. Multiple ACM's have been used for the extraction of a single region [4][5]. For instance in [4] two contours are used, one expands from inside the region and another contracts from the outside. This requires judicious initialization of the two contours. Some work has been done on the use of multiple ACMs for the extraction of multiple regions [6][7]. For example in [6] multiple regions are used but the approach is restricted to regions that have some common characteristic or property and weighting parameters are defined heuristically. In [7] several ACMs are initialized in the centers of divergence of the gradient vector flow field. Some of the centers are discarded using heuristic rules and the method is unable to deal with regions inside other regions.

We propose the use of multiple ACMs for the simultaneous extraction of multiple regions inspired in the Adaptive Snakes algorithm recently proposed in [9]. The multiple ACMs compete for the boundaries of the different regions. This competition alleviates the problem of contour initialization. The proposed method is able to deal with separate regions and also with nested regions. The method is based on the use of middle level features (strokes) instead of low level features (edge points) and the assignment of a weight to each stroke/model pair. Weight assignment is computed using the EM algorithm for MAP estimation.

This paper is organized as follows: section 2 formulates the problem, section 3 describes the proposed algorithm for multiple active contours, section 4 presents experimental results and section 5 concludes the paper.

\section{PROBLEM FORMULATION}

Let $y$ be the set of all edge points detected in an image and let us assume that $y$ is organized in connected components, called strokes, $y^{j}, j=1, \ldots, N$ where $y^{j}=\left\{y_{1}^{j}, \ldots, y_{n}^{j}\right\}$ is the set of edge points belonging to the $\mathrm{j}$-th stroke.

We will assume that the number of ACMs, L is known and we add an extra model to account for outliers. We designate it the outlier model. Let $x^{k}$ be the k-th active contour model, $k=1, \ldots, L$ defined by a sequence of 2D points $x_{i}^{k}, i=1, \ldots, M$;

$x^{k}$ can be either an open or a closed contour.

Figure 1 shows an example of an image with 3 strokes $y^{1}, y^{2}, y^{3}$ and two active contours $x^{1}$ and $x^{2}$.

We will assume that the strokes detected in the image are independent:

$p(y \mid x)=\prod_{j} p\left(y^{j} \mid x\right)$

and that each stroke is a mixture of $\mathrm{L}+1$ densities:

$$
p\left(y^{j} \mid x\right)=\sum_{k} \alpha_{k} p\left(y^{j} \mid x^{k}\right)
$$

where the $\alpha_{k}$ 's are the mixing proportions verifying $\alpha_{k} \geq 0$ and $\sum_{k} \alpha_{k}=1$.

Assuming a Gibbs distribution based on the Cohen potential we have [2] [9]:

$p\left(y^{j} \mid x^{k}\right)=\beta \exp \sum_{i} P\left(x_{i}^{k}, y^{j}\right)$

\footnotetext{
${ }^{1}$ This work was partially supported by FCT.
} 
where $P\left(x_{i}^{k}, y^{j}\right)$ is a potential function given by:

$$
P\left(x_{i}^{k}, y^{j}\right)=-\sum_{n} \phi_{\sigma}\left(\left|y_{n}^{j}-x_{i}^{k}\right|^{2}\right)
$$

where $\phi_{\sigma}\left(\left|y_{n}^{j}-x_{i}^{k}\right|^{2}\right)$ is the contribution of each feature $y_{n}^{j}$ to the potential and is obtained by convolving the edge map with the Gaussian kernel $\phi_{\sigma}(d)=\exp \left(-d / 2 \sigma^{2}\right)$. For the case of the outlier model, the contribution of each feature to the potential is constant, and chosen as $\phi_{\sigma}\left(|3 \sigma|^{2}\right)$.

Our aim is to estimate the L ACM's using the MAP criterion:

$$
\begin{aligned}
& x^{*}=\underset{x}{\arg \max _{x}} p(x \mid y) \\
& =\arg \max _{x}[\log p(y \mid x)+\log p(x)]
\end{aligned}
$$

Using (1) and (2) to solve (5) is equivalent to maximizing: $\log p(y \mid x)+\log p(x)$

$=\sum_{j} \log \left(\sum_{k} \alpha_{k} p\left(y^{j} \mid x^{k}\right)\right)+\log p(x)$

which can not be solved analytically. Therefore the EM algorithm is used, as detailed in section 3.

We adopt the following prior:

$\log p(x)=\sum_{k}\left(E_{\text {int }}\left(x^{k}\right)+\sum_{l \neq k} E_{\text {inter }}\left(x^{k}, x^{l}\right)\right)$

where $E_{\text {int }}\left(x^{k}\right)$ is a regularization energy that expresses the assumption that each contour is smooth and $E_{\text {inter }}\left(x^{k}, x^{l}\right)$ is another regularization energy that expresses the interaction between different active contours. For $E_{\text {int }}\left(x^{k}\right)$ we used:

$$
E_{\text {int }}\left(x^{k}\right)=\sum_{i}\left(\left\|x_{i}^{k}-x_{i-1}^{k}\right\|-l_{0}\right)^{2}
$$

where $l_{0}$ is the desired spacing between successive snake elements, set by the user. Setting $l_{0} \neq 0$ prevents the active contour from shrinking [8]. For $E_{\text {inter }}\left(x^{k}, x^{l}\right)$ we used:

$$
E_{\text {inter }}\left(x^{k}, x^{l}\right)=\sum_{i} \sum_{m} \varphi\left(\left|x_{m}^{l}-x_{i}^{k}\right|^{2}\right)
$$

In this expression $\varphi(d)$ is a distance measure that can be tailored to fit a particular application. Some examples for $\varphi(d)$ will be given in section 3.2.
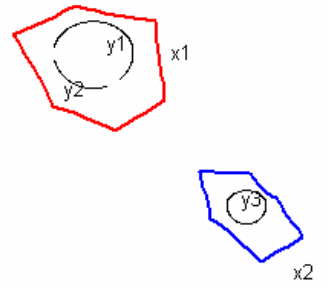

Figure 1 Image with 3 strokes and two active contours.

\section{MULTIPLE ACTIVE CONTOURS}

The EM algorithm assumes that $\mathrm{y}$ is incomplete data and that the complete data includes binary labels $z_{j}, j=1, \ldots, N$ with $z_{j}=\left\{z_{j}^{1}, \ldots, z_{j}^{L+1}\right\}$, that indicate which model generated the stroke, $z_{j}^{k}=1$ means that stroke $y^{j}$ was generated by model $x^{k}$. The complete log likelihood is given by:

$\log p(y, z \mid x)=\sum_{j} \sum_{k} z_{j}^{k} \log p\left(y^{j} \mid x^{k}\right)$

Instead of maximizing (5), the EM algorithm alternates between two steps. In the E-step it finds the conditional expectation of the complete log likelihood with respect to the unknown $\mathrm{x}$ given the observed data $\mathrm{y}$ and the current estimate, $\hat{x}$.

$Q(x, \hat{x})=E[\log p(y, z \mid x) \mid y, \hat{x}]$

$Q(x, \hat{x})=E\left[\sum_{j} \sum_{k} z_{j}^{k} \log \left[\alpha_{k} p\left(y^{j} \mid x^{k}\right)\right]\right]$

$=\sum_{j} \sum_{k} w_{k}^{j} \log \left[\alpha_{k} p\left(y^{j} \mid x^{k}\right)\right]$

where $w_{k}^{j}$ is set of weights summing to one assigned to each stroke. Each weight $w_{k}^{j}$ represents the soft assignment of stroke $y^{j}$ to the $x^{k}$ active contour. The weights are given by:

$w_{k}^{j}=p\left(z_{j}^{k}=1 \mid y^{j}, \hat{x}\right)=\frac{\alpha_{k} p\left(y^{j} \mid x^{k}\right)}{\sum_{m} \alpha_{m} p\left(y^{j} \mid x^{m}\right)}$

In the M-step the estimation of the active contour is obtained by the maximization of:

$U(x, \hat{x})=Q(x, \hat{x})+\log p(x)$

The algorithm sequentially performs one $\mathrm{E}$ step and one $\mathrm{M}$ step for each of the ACMs in a predefined order and iterates until convergence. This methodology is called the Component 
Wise EM (CEM) and it's converge was shown in [11]. The E and $\mathrm{M}$ steps will be detailed in the following sections.

\subsection{The E-Step}

In the E-step the weights are calculated. Substituting (3) into (13) we obtain the following expression:

$$
w_{k}^{j}=\frac{\alpha_{k} \prod_{i} \exp \left(\sum_{n} \phi_{\sigma}\left(\left|y_{n}^{j}-x_{i}^{k}\right|^{2}\right)\right.}{\sum_{m} \alpha_{m} \prod_{i} \exp \left(\sum_{n} \phi_{\sigma}\left(\left|y_{n}^{j}-x_{i}^{m}\right|^{2}\right)+\alpha_{L+1} \exp \left(N^{j} M \phi_{\sigma}\left(|3 \sigma|^{2}\right)\right)\right.}
$$

where the last term of the denominator is the contribution of the outlier model.

The mixing proportions are updated by:

$$
\alpha_{k}=\sum_{j} w_{k}^{j}
$$

\subsection{The M-Step}

In the M-step the estimation of the active contour is obtained by the minimization of (12) performed by the gradient algorithm:

$x_{t+1}^{k}=x_{t}^{k}-\gamma \nabla_{x}(Q(x, \widehat{x}))$

where $\nabla_{x}$ represents the gradient. This equation can be rewritten as follows:

$$
x_{t+1}^{k}=x_{t}^{k}-\gamma_{\text {int }} f_{\text {int }}-\gamma_{\text {ext }} f_{\text {ext }}-\gamma_{\text {inter }} f_{\text {inter }}
$$

where $f_{\text {ext }}\left(x_{i}^{k}\right), f_{\text {int }}\left(x_{i}^{k}\right)$ and $f_{\text {inter }}\left(x_{i}^{k}\right)$ are external, internal and interaction forces. External and internal forces are given by expressions (19) and (20):

$$
\begin{aligned}
& f_{\text {ext }}\left(x_{i}^{k}\right)=-\frac{1}{\sigma^{2}} \sum_{j} w_{j}^{k} \sum_{n}\left(y_{n}^{j}-x_{i}^{k}\right) \phi_{\sigma}\left(\left|y_{n}^{j}-x_{i}^{k}\right|^{2}\right) \\
& f_{\text {int }}\left(x_{i}^{k}\right)=-2\left(\frac{l_{i-1}-l_{0}}{l_{i-1}}\left(x_{i}^{k}-x_{i-1}^{k}\right)+\frac{l_{i+1}-l_{0}}{l_{i+1}}\left(x_{i}^{k}-x_{i+1}^{k}\right)\right)
\end{aligned}
$$

where $l_{i-1}=\left\|x_{i}^{k}-x_{i-1}^{k}\right\|$ and $l_{i+1}=\left\|x_{i}^{k}-x_{i+1}^{k}\right\|$.

The expression of the interaction force depends on the choice of $\varphi(d)$ in equation (7). For instance, if we expect each model to attract the other models, we can use $\varphi(d)=-\exp \left(-d / 2 \sigma_{\text {inter }}^{2}\right)$ leading to:

$$
f_{\text {inter }}\left(x_{i}^{k}\right)=\frac{1}{\sigma_{\text {inter }}^{2}} \sum_{l \neq k} \sum_{m}\left(x_{i}^{k}-x_{m}^{l}\right) \varphi\left(\left|x_{m}^{l}-x_{i}^{k}\right|^{2}\right)
$$

Similarly, we can define repulsion forces between models by using $\varphi(d)=\exp \left(-d / 2 \sigma_{\text {inter }}^{2}\right)$ leading to:
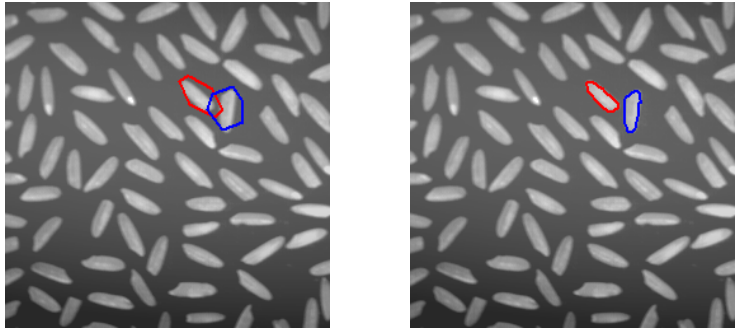

Figure 2 Results obtained with the proposed method using two ACMs; initial contours and contour estimates at iteration 2.

$$
f_{\text {inter }}\left(x_{i}^{k}\right)=\frac{1}{\sigma_{\text {inter }}^{2}} \sum_{l \neq k} \sum_{m}\left(x_{m}^{l}-x_{i}^{k}\right) \varphi\left(\left|x_{m}^{l}-x_{i}^{k}\right|^{2}\right)
$$

Another possibility is to define $\varphi(d)$ in order to impose some kind of geometrical constraint between the different active contours. For instance for concentric models we can use the distance between the models centroids:

$$
f_{\text {inter }}\left(x_{i}^{k}\right)=\sum_{i} x_{i}^{k}-\frac{1}{L-1} \sum_{l \neq k} \sum_{m} x_{m}^{l}
$$

This method belongs to a class of constrained clustering algorithms of methods, denoted Unified Framework, in which models points are attracted towards the centroids $\xi_{i}^{k}$ of their attraction regions [10]. Therefore the external forces can be rewritten as:

$$
f_{\text {ext }}\left(x_{i}^{k}\right)=\mu_{i}^{k}\left(\xi_{i}^{k}-x_{i}^{k}\right)
$$

with

$$
\mu_{i}^{k}=\sum_{j} w_{j}^{k} \sum_{n} \phi_{\sigma}\left(\left|y_{n}^{j}-x_{i}^{k}\right|^{2}\right)
$$

and

$$
\xi_{i}^{k}=\frac{\sum_{j} w_{j}^{k} \sum_{n} y_{n}^{j} \phi_{\sigma}\left(\left|y_{n}^{j}-x_{i}^{k}\right|^{2}\right)}{\mu_{i}^{k}}
$$

\section{EXPERIMENTAL RESULTS}

This section presents examples to illustrate the performance of the proposed method. The examples were performed in the following conditions. Edges were obtained with the Canny edge detector and strokes were obtained with a connected components labeling algorithm. Strokes with length smaller than a predefined threshold $l_{\text {min }}$ were considered noise and discarded. The external forces acting on each model unit were multiplied by independent gains as suggested in [10]:

$$
\left(\gamma_{\text {ext }}\right)_{i}^{k}=\frac{1}{\mu_{i}^{k}} \min \left(1, \frac{\Delta_{\max }}{\left|\xi_{i}^{k}-x_{i}^{k}\right|}\right)
$$


where $\Delta_{\max }$ is the maximum displacement of the model units in each iteration. All the experiments used $\Delta_{\max }=10$ and the gain factors $\gamma_{\text {int }}$ and $\gamma_{\text {inter }}$ were chosen manually.

The first example illustrates the performance of the algorithm in the presence of multiple objects (grains of rice). Figure 2 shows the initial contours on the left and the final contours on the right. It can be seen that although the initial contours were overlapping the final result was able to separate two different rice grains and to discard the other grains classifying them as outliers.

The second example shows the performance of the proposed algorithm with more than two elastic models. It also shows the robustness with respect to initialization since all the contours were initialized in the same way. In this example four closed ACMs were used to segment the different rings of the eyespot of a butterfly wing. Figure 3 shows the initial contours on the left and the final contours on the right. Although the ACMs were equally initialized each converged to a different ring of the butterfly eyespot. The third example illustrates the application of the algorithm to medical data ${ }^{2}$. In this example three closed ACMs were used to segment the skull and the brain in a MRI medical image. All the ACMs were initialized with the same contour. Figure 4 shows the initial contours on the left and the final contours on the right. Although all ACM's were equally initialized two of them converged towards the exterior skull borders and another converged towards the outline of the brain.

We emphasize that it is because of the competition between models and the use of the CEM algorithm that different models converge to different strokes even when they are initialized with the same configuration.

\section{CONCLUSIONS}

This paper presents an algorithm for the extraction of multiple regions using multiple active contour models (ACMs) which also accounts for outlier features detected in the image. It is shown that the proposed algorithm is able to robustly estimate all the deformable contours and to compute the association probability between strokes and multiple models. In addition, it can deal with separate regions as well as nested regions and it is less sensitive to the initial configuration than the conventional single ACMs.

Future work should consider the automatic estimation of the number of multiple models as well as automatic initialization procedures.

\section{REFERENCES}

[1] Kass, M.; Witkin, A. and Terzopoulos, D., "Snakes: Active contour models", International Journal of Computer Vision. 1(4): 321-331, 1987.

[2] L. Cohen, "On active contour models and balloons", CVGIP: Image Understanding, 53(2): 212-218, 1991.

\footnotetext{
${ }^{2}$ The authors would like to thank IBEB and CDRM for providing the MRI images.
}
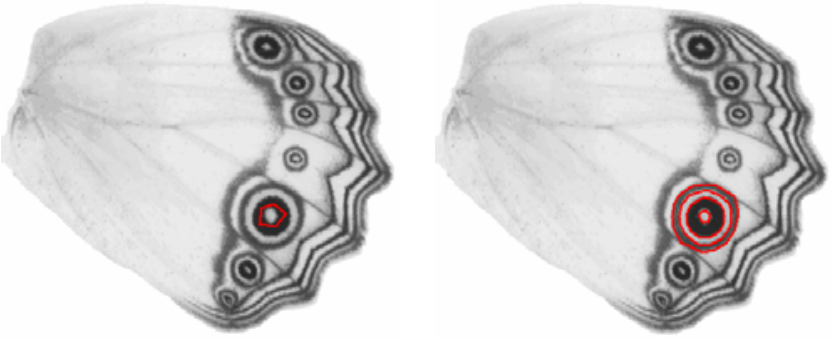

Figure 3 Results obtained with the proposed method using four ACMs; Initial contours and contour estimates at iteration 5.
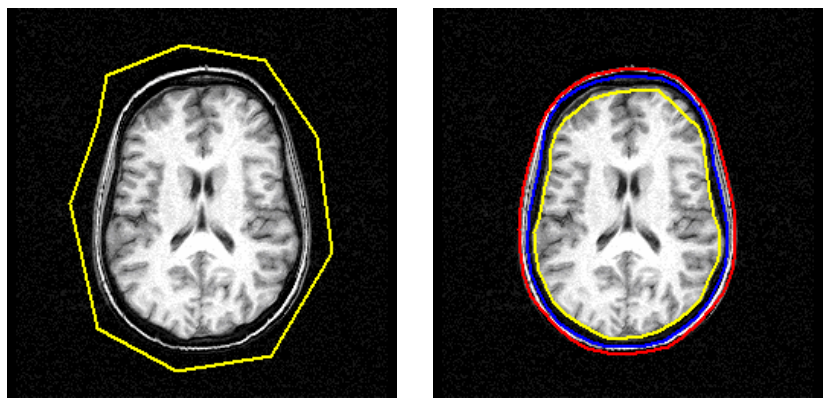

Figure 4 Results obtained with the proposed method using three ACMs; initial contours and contour estimates at iteration 3.

[3] Xu, C. and Prince, J. L., "Snakes, shapes, and gradient vector flow”, IEEE Trans. on Image Processing, 7(3):359-369, 1998.

[4] Gunn, S. R. and Nixon, M. S., "A robust snake implementation: A Dual Active Contour”, IEEE Trans. on Pattern Analysis and Machine Intelligence, 19(1): 63-68, 1997. [5] Chalana, V.; Linker, D.T.; Haynor, D.R. and Yongmin Kim, "A multiple active contour model for cardiac boundary detection on echocardiographic sequences", IEEE Trans. on Medical Imaging, 13(3): 290-298, 1996.

[6] Srinark, T. and Kambhamettu, C., "A framework for multiple snakes", Proceedings of the 2001 IEEE Computer Society Conference on Computer Vision and Pattern Recognition, II: 202-209, 2001.

[7] Xingfei, G and Jie, T.; "An automatic active contour model for multiple objects", Proceedings of the 16th International Conference on Pattern Recognition, 2: 881-884, 2002.

[8] Leymarie, F.; Levine, M.D., "Tracking Deformable Objects in the Plane Using an Active Contour Model”, IEEE Trans. on Pattern Analysis and Machine Intelligence, 15(6): 617-634, 1993.

[9] Nascimento, J. C. and Marques, J. S., "An adaptive potential for robust shape estimation", Image and Vision Computing, 21(13-14): 1107-1116, 2003.

[10] Abrantes, A. J. and Marques, J. S., "A class of constrained clustering algorithms for object boundary extraction", IEEE Transactions on Image Processing, 5(11): 1507-1521, 1996. [11] Celeux, G., Chréetien, Forbes, F. and Mkhadri, A. "A component-wise EM algorithm for mixtures", Journal of Computational and Graphical Statistics, 10: 699-712, 2001. 